\title{
HS-SPME-GCMS Method Development for Sensitive Diisopropylphenol Analysis Suited for Adsorption/Absorption Studies and Cleaning Validations
}

\author{
K. E. PICKL ${ }^{1}$, V. AdAMEK ${ }^{2}$, R. GORges ${ }^{2}$, F. M. SinNeR ${ }^{1}$ \\ ${ }^{1}$ Institute of Medical Technologies and Healthmanagement, JOANNEUM RESEARCH, Graz, Austria \\ ${ }^{2}$ Research Center for Pharmaceutical Engineering, Graz, Austria \\ E-mail: karin.pickl@joanneum.at (K. E. Pickl)
}

Sci Pharm. 2010; 78: 698

doi:10.3797/scipharm.cespt.8.PPAT09

The interaction of active pharmaceutical ingredients with various surfaces and solutions are of high interest in pharmaceutical industry in particular with respect to development of new formulations, new packaging material [1] and cleaning validation of production plants [2]. Experimental adsorption/absorption studies as well as the performance of cleaning validations provide valuable data for these research fields; however appropiate analytical methods are needed for the drug of interest. In the case of diisopropylphenol - a small lipophilic drug which is typically formulated as lipid emulsion for intravenous injection - the main analytical challenge is a highly sensitive method (in the $\mathrm{ng} / \mathrm{ml}$ range) suitable to be applied to a variety of different sample matrices (lipid emulsions, aqueous solutions, organic solvents as well as various surfaces). In particular, the analysis of diisopropylphenol in complex matrices such as lipid formulations requires efficient samplecleanup methods. We here report the development and evaluation of a new headspace-solid phase microextraction (HS-SPME) methodology for GC-MS as a cleanup procedure for the moderately volatile drug diisopropylphenol in various matrices. Optimization of the HS-SPME procedure with respect to temperature, incubation times and matrix was carried out and linear calibration curves in the range of $2-200 \mathrm{ng} / \mathrm{ml}$ could be achieved for various matrices including lipid emulsions.

This work was supported by the RCPE K1 Competence Center - Initiated by the Federal Ministry of Transport, Innovation \& Technology (MMVIT) and the Federal Ministry of Economics \& Labour (BMWA). Funded by FFG, Land Steiermark and Steirische Wirtschaftsförderung (SFG).

[1] FDA Guidance for Industry Container Closure Systems for Packaging Human Drugs and Biologics, May 1999.

[2] United States, Code of Federal Regulation, Titel 21 Food and Drugs, Part 211 CURRENT GOOD MANUFACTURING PRACTICE FOR FINISHED PHARMACEUTICALS, § 211.67 equipment cleaning and maintenance. 